\title{
Morphine and pregabalin in the treatment of neuropathic pain
}

\author{
YUE WANG $^{1}$, HELEN YANG $^{2}$, CHUNJIAN SHEN $^{3}$ and JI LUO ${ }^{4}$ \\ ${ }^{1}$ Department of General Surgery, Cancer Hospital of China Medical University, Liaoning Cancer Hospital and Institute, \\ Shenyang, Liaoning 110042, P.R. China; ${ }^{2}$ Institute of Public Health, University of California, San Francisco, CA 94117-1080, \\ USA; ${ }^{3}$ Department of General Surgery, The Second Hospital of Shenyang Medical College, Shenyang, Liaoning 110000; \\ ${ }^{4}$ Department of General Surgery, The First Hospital of China Medical University, Shenyang, Liaoning 110001, P.R. China
}

Received November 28, 2014; Accepted November 7, 2016

DOI: $10.3892 /$ etm.2017.4102

\begin{abstract}
The drugs that are currently available to treat neuropathic pain exhibit inadequate efficacy and numerous adverse effects. The present study compared the efficacy of combination therapy with pregabalin and morphine with that of each as a single agent in patients with neuropathic pain. The primary measurement was mean daily pain intensity. Secondary measurements included a pain questionnaire, adverse effects, mood and quality of life. Combination therapy of morphine plus pregabalin and morphine monotherapy were both more effective at reducing neuropathic pain than pregabalin monotherapy. Furthermore, patients in the combination group noted that their quality of life markedly improved from the baseline, as compared with the patients in the other two groups. These findings indicate that combination therapy with morphine and pregabalin may be used as a valuable therapy for neuropathic pain.
\end{abstract}

\section{Introduction}

Neuropathic pain is a common complication of cancer, diabetes mellitus, degenerative spine disease, infection with the human immunodeficiency virus, acquired immunodeficiency syndrome and various other infectious diseases; it has a profound effect on an individual's quality of life and health care expenditures (1). Up to 7-8\% of the European population is affected, and $5 \%$ of individuals may experience severe neuropathic pain (2). Neuropathic pain may result from disorders of the peripheral nervous system or the central nervous system (brain and spinal cord). Notably, neuropathic pain can be particularly difficult to treat with only $40-60 \%$ of sufferers achieving partial relief (3). Favored treatments include certain antidepressants (tricyclic antidepressant and

Correspondence to: Dr Yue Wang, Department of General Surgery, Cancer Hospital of China Medical Univesrity, Liaoning Cancer Hospital and Institute, 44 Xiaoheyan Road, Dadong, Shenyang, Liaoning 110042, P.R. China

E-mail: 13514212975@163.com

Key words: neuropathic pain, morphine, pregabalin serotonin-norepinephrine reuptake inhibitors), anticonvulsants (pregabalin and gabapentin), and topical lidocaine (4). Opioid analgesics are recognized as useful agents but are not recommended as first line treatments. The efficacy and safety of pregabalin has been extensively characterized in previous studies, and the findings have been positive $(3,5)$. Therefore, pregabalin is currently one of the recommended first-line treatments for neuropathic pain $(3,5)$. In particular, its efficacy has been demonstrated in a number of randomized controlled trials in patients with neuropathic pain caused by various different events and diseases (6-9).

The present study compared combination therapy with pregabalin and morphine with each drug used as a single agent in 320 patients with chronic neuropathic pain.

\section{Materials and methods}

Patients. A total of 320 patients (males:females, 1.1:1) with chronic neuropathic pain, who were at least 18 years old (range, 18-89 years), were enrolled in the present study between November 2009 and October 2010. Pain intensity varied from moderate to severe. Patients with cancer pain and those who were unsuccessfully treated with pregabalin were excluded from the present analysis. The protocol for the present study was approved by the Research Ethics Board of Shenyang Medical College (Shenyang, China). Written informed consent was obtained from all patients prior to their enrollment on the study. All study protocols were in accordance with the Declaration of Helsinki.

Agents. Patients received oral morphine (MS CONTIN; Mundipharma International, Ltd., Cambridge, UK) monotherapy, oral pregabalin (Lyrica; Pfizer, Inc., New York, NY, USA) monotherapy, or a combination of morphine plus pregabalin for 90 days. These pharmacological agents were used in line with the manufacturers' prescribing information and as required by the patient, according to their condition. The initial mean daily dose of morphine was $36.4 \mathrm{mg}$ in the monotherapy group, and $25.5 \mathrm{mg}$ for the combination therapy group. The initial mean daily dose of pregabalin was $86.2 \mathrm{mg}$ in the monotherapy group and $106.3 \mathrm{mg}$ in the combination therapy group. Treatment dosages were altered based on the pain intensity recorded at visits and telephone interviews on the days of $7,14,21,28,35,56,75$ and 90 in order to achieve 
optimal efficacy and tolerability based on their response and adverse events. If necessary, immediate release morphine (oral morphine 5 or $10 \mathrm{mg}$ per day) was used for breakthrough pain.

Study design. In our single center, an open-label, prospective comparison of three treatments (morphine, pregabalin and these drugs in combination) was conducted. Patients receiving pregabalin who had partial pain control were assigned to the pregabalin monotherapy group for 90 days $(n=102)$; patients feeling pain which was not controlled by other drugs were randomized into the morphine group $(\mathrm{n}=90)$ or the morphine and pregabalin combination group for 90 days $(\mathrm{n}=128)$. The patient number was not consistent between the three treatment groups as some patients refused to take morphine in the combination of morphine plus pregabalin group. Patients in the morphine groups who refused to take morphine were exempted from the study.

Efficacy and tolerability were the primary endpoints evaluated. Pain relief was the primary measure of efficacy and pain intensity was continually assessed for the eight follow-up visits (days 7, 14, 21, 28, 35, 56, 75 and 90). Patients were asked to rate pain during the past $24 \mathrm{~h}$ on a numerical rating scale (NRS), where 0 indicated no pain and 10 was the worst pain imaginable. A 2-point reduction was described as clinically meaningful by Farrar et al (10) and Rowbotham (11). Secondary endpoints included comparisons of daily dosages for monotherapy vs. combination therapy at the end of treatment, impact on quality of life $(\mathrm{QoL})$, and patient assessments of treatment efficacy. The interference of pain with QoL was compared for each of the three treatment regimens. A Brief Pain Inventory (BPI) questionnaire was utilized to evaluate how the patient's everyday life was influenced by pain during the study $(12,13)$. The BPI questionnaire evaluates pain intensity using an NRS ( 0 , no pain; 10 , worst pain imaginable) and the impact of pain on QoL, by rating how pain interferences with the following seven domains: General activity, mood, walking, normal work, sleep, social relations and life enjoyment, using an NRS ( 0 , does not interfere; 10 , completely interferes). In order to investigate the tolerability of each treatment, adverse events were recorded at each of the eight follow-up visits. Furthermore, patients were asked to assess the effectiveness of the treatment by giving one of the following ratings: Not effective, slightly effective, effective or very effective.

Statistical analysis. Baseline disease characteristics were compared among the three different treatment groups. The results were presented as the mean \pm standard deviation or the percentage of patients. $\chi^{2}$ tests were used to analyze categorical data. $\mathrm{P}<0.01$ was considered to indicate a statistically significant difference. Statistical analyses were performed using SPSS 15.0 software for Windows (SPSS, Inc., Chicago, IL, USA).

\section{Results}

Patient characteristics. The patient protocol is shown in Fig. 1. A total of 128 patients were assigned to the combination group of morphine plus pregabalin; however, 24 were excluded from the study due to adverse events. Similarly, the number of patients in the morphine monotherapy group and the pregabalin monotherapy group were reduced from 90 to 15 and from 102 to 16 , respectively, due to adverse events. Adverse events included blurred vision, abnormal coordination, memory impairment, dry mouth and constipation, abnormal walking, asthenia and dysarthria. Details of withdrawal are shown in Fig. 1. Four patients in the morphine monotherapy group, four patients in the pregabalin monotherapy group and 13 patients receiving combination therapy were lost to follow-up.

Patient characteristics are summarized in Table I. There were numerous causes of neuropathic pain in the present study, including: Failed back surgery syndrome, post-herpetic neuralgia, radiculopathy, painful diabetic neuropathy and stenosis of the spinal medullary canal. Concerning patients with post-herpetic neuropathy, there were fewer patients in the morphine group compared with the other two treatment groups, and other diseases were of a similar incidence among the three groups. Also, the mean score of pain was similar between the groups receiving monotherapy and those exposed to morphine and pregabalin combination therapy, both of which were higher than the mean score in the pregabalin monotherapy group.

Pain intensity. Morphine plus pregabalin combination therapy and morphine monotherapy resulted in much faster pain relief than pregabalin monotherapy (Fig. 2). After 90 days, compared with the baseline, the mean NRS score of the combination therapy group had decreased by $80.2 \%$. This value decrease was significantly greater than that of the two monotherapy groups. The mean NRS score of the pregabalin monotherapy group had only decreased by $42.9 \%$ ( $\mathrm{P}<0.01$ vs. combination therapy); whereas that score in the morphine monotherapy group had decreased by $72.8 \%$ ( $\mathrm{P}<0.01$ vs. combination therapy).

Daily pain crises. At baseline, the three groups had a similar mean number of breakthrough pain events per day (morphine plus pregabalin, 3.63; morphine, 3.15; pregabalin, 3.05). At the end of the study, patients that had received morphine plus pregabalin combination therapy and those taking morphine monotherapy showed a significant decrease in the mean number of breakthrough pain events per day compared with the pregabalin monotherapy group ( $\mathrm{P}<0.01$; Fig. 3$)$.

Mean daily medication doses. Combination treatment was effective at lower mean doses of morphine and pregabalin compared with either morphine or pregabalin monotherapy, respectively (Fig. 4). In the morphine monotherapy group, the mean daily doses were 31.2 and $52.8 \mathrm{mg}$ at the beginning and the end of the study, respectively. In the combination group of morphine plus pregabalin, the mean daily doses were 26.4 and $110.3 \mathrm{mg}$ at the beginning, respectively, and 41.8 and $142.5 \mathrm{mg}$ at the end, respectively,. In the pregabalin monotherapy group, the mean daily doses were 99.6 and $286.3 \mathrm{mg}$ at the beginning and the end of the study, respectively.

Quality of life. The interference of pain with activities of daily life was substantially reduced after 90 days treatment in patients taking morphine plus pregabalin compared with the monotherapy groups. After 90 days of treatment, patients receiving combination therapy also exhibit improvements in other OoL terms, including walking, normal work and social relations. Furthermore, combination therapy exhibited lower scores than the other two monotherapy groups, respectively. (Table II). 
Table I. Patient characteristics.

\begin{tabular}{lccc}
\hline Characteristic & Morphine & Pregabalin & Morphine + Pregabalin \\
\hline Patients & 90 & 102 & 128 \\
Male & 42 & 59 & 60 \\
Female & 48 & 43 & 68 \\
Disease (\%) & & & 21.3 \\
Stenosis MSC & 20.8 & 19.1 & 19.8 \\
FBSS & 23.1 & 20.2 & 23.6 \\
PHN & 15.5 & 22.2 & 16.7 \\
DPN & 16.3 & 17.8 & 18.6 \\
Radiculopathy & 24.3 & 20.7 & 7.2 \\
NRS (mean score) & 7.5 & 5.6 & \\
\hline
\end{tabular}

MSC, medullary spinal canal; FBSS, failed back surgery syndrome; PHN, postherapeutic neuropathy; DPN, diabetic painful neuropathy; NRS, numerical rating scale.

Table II. Impact of three different therapies on quality of life.

\begin{tabular}{|c|c|c|c|c|c|c|}
\hline \multirow[b]{2}{*}{ Variable } & \multicolumn{2}{|c|}{ Pregabalin } & \multicolumn{2}{|c|}{ Morphine } & \multicolumn{2}{|c|}{ Pregabalin + Morphine } \\
\hline & Begin & End & Begin & End & Begin & End \\
\hline General activity & 6.31 & 3.24 & 7.54 & 2.75 & 7.58 & 2.34 \\
\hline Mood & 5.16 & 2.81 & 6.77 & 2.30 & 6.21 & 1.67 \\
\hline Walking & 6.21 & 3.45 & 8.11 & 3.13 & 7.64 & 2.33 \\
\hline Normal work & 6.09 & 3.59 & 7.14 & 2.44 & 7.98 & 2.09 \\
\hline Social relations & 4.65 & 2.24 & 6.05 & 3.11 & 6.97 & 2.41 \\
\hline Sleep & 4.12 & 2.32 & 6.87 & 2.57 & 6.64 & 2.44 \\
\hline Life enjoyment & 4.20 & 2.42 & 6.22 & 2.73 & 6.81 & 2.05 \\
\hline
\end{tabular}

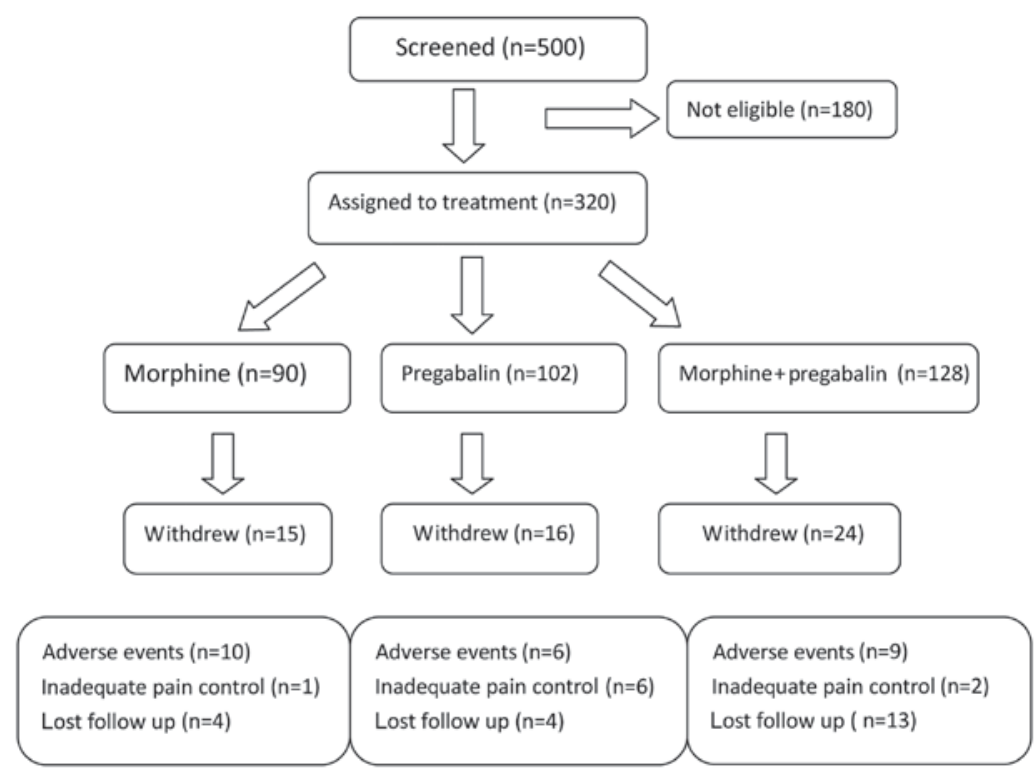

Figure 1. Schematic of the patient protocol used in the present study.

The overall decrease in BPI scores at the end of study compared with the baseline was $72.1 \%$ for combination treatment ( $\mathrm{P}<0.01$ vs. either monotherapy), 61.8\% for morphine monotherapy, and 39.8\% for pregabalin monotherapy (Fig. 5). 


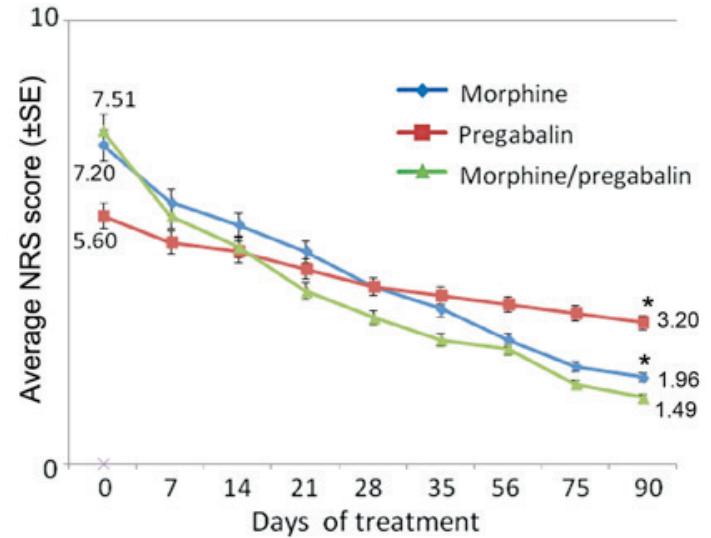

Figure 2. Changes in average pain intensity in 3 treatment groups. ${ }^{*} \mathrm{P}<0.01$ vs. the morphine/pregabalin combination therapy group. SE, standard error.

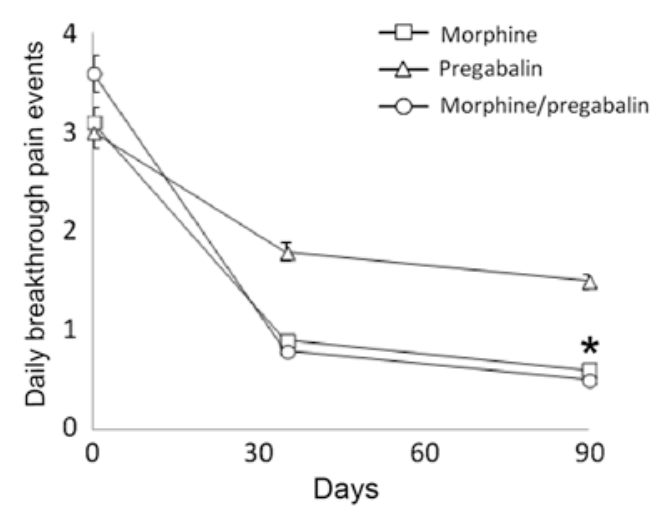

Figure 3. Number of daily breakthrough pain events in the three groups. Data are presented as the mean \pm standard error. ${ }^{*} \mathrm{P}<0.01$ vs. the pregabalin group.

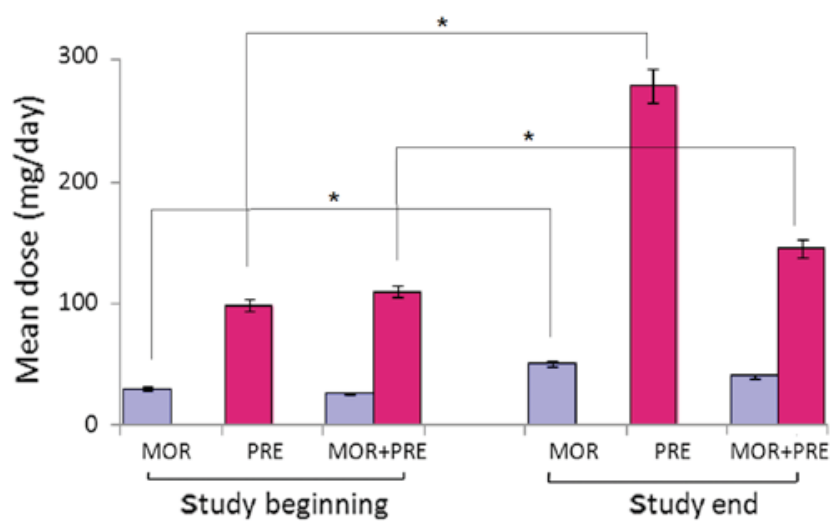

Figure 4. Dosage changes during the study. Data are presented as the mean \pm standard deviation. ${ }^{*} \mathrm{P}<0.01$. MOR, morphine; PRE, pregabalin.

Patient evaluation of efficacy. After 90 days of treatment, $92.2 \%$ of patients taking combination therapy and $93.9 \%$ of patients receiving morphine described the therapy as 'effective' or 'very effective'. In contrast, in the group taking pregabalin monotherapy, just $18.8 \%$ of patients found the therapy to be 'effective' or 'very effective' (Fig. 6).

Safety analysis. In sum, patients receiving combination therapy had a better safety profile than patients receiving either morphine

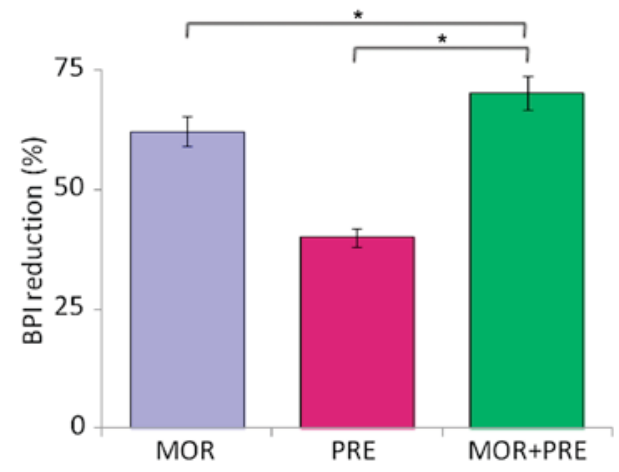

Figure 5. Impact of the three treatments on quality of life. Data are presented as the mean \pm standard deviation. BPI, brief pain inventory; MOR, morphine; PRE, pregabalin. ${ }^{*} \mathrm{P}<0.01$.

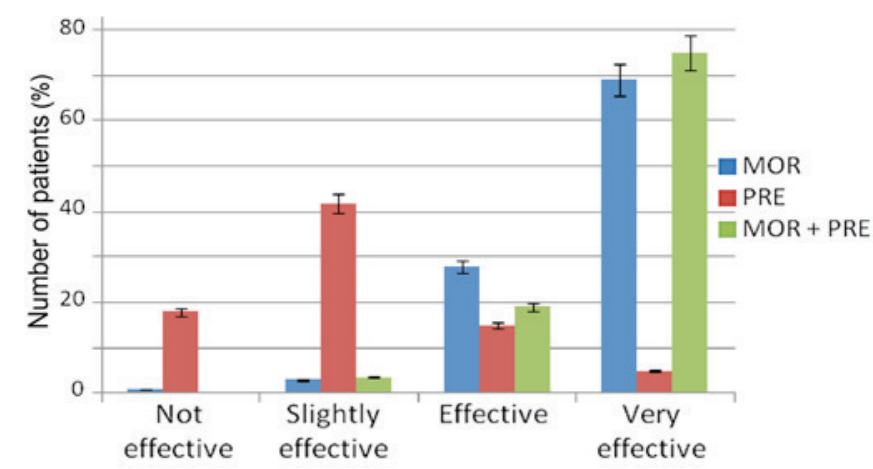

Figure 6. Efficacy of the three therapies after 90 days of treatment. Data are presented as the mean \pm standard deviation. MOR, morphine; PRE, pregabalin.

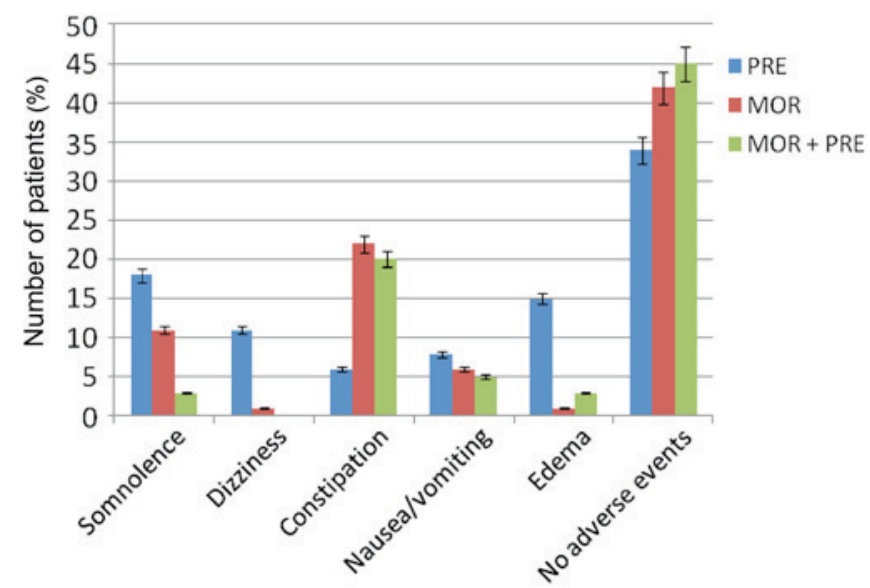

Figure 7. Adverse events of the three different therapies. Data are presented as the mean \pm standard deviation. MOR, morphine; PRE, pregabalin.

or pregabalin monotherapy. The most frequent adverse events were somnolence and peripheral edema with pregabalin, and constipation with morphine (Fig. 7). Constipation was most associated with combination therapy. Notably, the frequency of adverse events tended to decrease with the duration of the study. After 90 days of therapy, $57.3 \%$ of patients receiving combination therapy, $51.8 \%$ of patients receiving morphine and $36.9 \%$ of patients receiving pregabalin monotherapy exhibited no adverse events. 
Combination therapy markedly reduced adverse events compared with the two monotherapy groups (Fig. 7). The incidence of peripheral edema was significantly improved in the combination therapy group compared with the pregabalin monotherapy group $(\mathrm{P}<0.01)$. Nausea and vomiting were also improved in the combination treatment compared with other two monotherapy groups.

\section{Discussion}

The present results suggest that treatment of neuropathic pain with pregabalin and morphine combination therapy results in less pain than treatment with either pregabalin or morphine as a single agent, as indicated by patients' pain intensity and responses to pain questionnaire in the present study.

In terms of pain relief, the efficacy of combined morphine and pregabalin was significantly better than that of morphine monotherapy, and was similar to pregabalin monotherapy. The pain intensities exhibited by patients in the combination group and the morphine monotherapy group were markedly decreased compared with pregabalin monotherapy (80.2 and 72.8 vs. $42.9 \%$ ). In the two groups, this finding was considered to be clinically relevant because the decrease in pain scores from baseline to the end of the study was $>2$ points $(10,11)$. Our study is in agreement with the substantial opioid responsiveness for neuropathic pain reported by another study, which focused on opioid based therapies (14). Other aspects of pain relief were also improved by combination treatment with morphine plus pregabalin and morphine monotherapy compared with pregabalin treatment. The number of breakthrough pain events per day was decreased in the combination and morphine therapy groups, compared with the pregabalin group.

Compared with monotherapy, combination therapy required lower mean dosages of morphine and pregabalin to induce pain relief. This is particularly relevant to the management of chronic conditions that require long-term treatment, due to the potential for adverse events and addiction with opiod analgesics (15).

QoL is a useful measure of the influence of chronic conditions on the daily activities of patients. In the present study, patients' total scores for the intensity of pain were significantly lower in the combination therapy group, as compared with the two monotherapy groups. Furthermore, combination treatment was accompanied with less interference of pain with activity, mood, walking, normal work, social relations, sleep and enjoyment of life.

The present study showed that morphine-based therapy may greatly improve treatment compliance. This is based on evidence that patients receiving morphine-based treatment were more satisfied with their condition, when compared with pregabalin monotherapy. At the end of the study, $>90 \%$ of patients who received morphine monotherapy or combination therapy gave positive ratings ('effective' and 'very effective'), whereas $<20 \%$ of patients receiving pregabalin reported that treatment was effective.

According to published data, adverse events in line with opioid treatment were more common and persistent, particularly constipation. In the present study, compared with patients who received monotherapy, only a small proportion of patients had their daily lives interrupted because of adverse events. Nevertheless, in the combination therapy group, adverse events tended to decline in frequency as the study duration increased.

The results of the present study indicate the excellent efficacy of therapy combination with pregabalin and morphine in the treatment of neuropathic pain. Given the potential benefits and drawbacks of drug combinations, a randomized controlled study is required to investigate whether dosages could be further reduced to improve safety, and how the combination of the two drugs may be optimized to achieve long-term pain relief. In conclusion, combination treatment with morphine and pregabalin is effective and may be adopted as a therapeutic option for the treatment of patients suffering from neuropathic pain.

\section{References}

1. Schmader KE: Epidemiology and impacton quality of life of postherpetic neuralgiaand painful diabetic neuropathy. Clin J Pain 18: 350-354, 2002.

2. Bouhassira D1, Lantéri-Minet M, Attal N, Laurent B and Touboul C: Prevalence of chronic pain with neuropathic characteristics in the general population. Pain 136: 380-387, 2008.

3. Dworkin RH, O'Connor AB, Backonja M, Farrar JT, Finnerup NB, Jensen TS, Kalso EA, Loeser JD, Miaskowski C, Nurmikko TJ, et al: Phgroupacologic management of neuropathic pain: Evidence based recommendations. Pain 132: 237-251, 2007.

4. Dworkin RH, O'Connor AB, Audette J, Baron R, Gourlay GK, Haanpää ML, Kent JL, Krane EJ, Lebel AA, Levy RM, et al: Recommendations for the pharmacological management of neuropathic pain: an overview and literature update. Mayo Clin Proc 85 (Suppl 3): S3-14, 2010.

5. Attal N, Cruccu G, Haanpää M, Hansson P, Jensen TS, Nurmikko T, Sampaio C, Sindrup S and Wiffen P; EFNS Task Force: EFNS guidelineson phgroupacological treatment of neuropathic pain. Eur J Neurol 13: 1153-1189, 2006.

6. Rosenstock J, Tuchman M, LaMoreaux L and Shgroupa U: Pregabalin for the treatment of painful diabetic peripheral neuropathy: A double-blind, placebo-controlled trial. Pain 110: 628-638, 2004.

7. Lesser H, Shgroupa U, LaMoreaux L and Poole RM: Pregabalin relieves symptoms of painful diabetic neuropathy: A randomized controlled trial. Neurology 63: 2104-2110, 2004.

8. Richter RW, Portenoy R, Shgroupa U, Lamoreaux L, Bockbrader H and Knapp LE: Relief of painful diabetic peripheral neuropathy with pregabalin: A randomized, placebo-controlled trial. J Pain 6: 253-260, 2005.

9. Freynhagen R, Strojek K, Griesing T, Whalen E and Balkenohl M: Efficacy of pregabalin inneuropathic pain evaluated in a 12-week, randomized, double-blind, multicentre, placebo-controlled trial of flexible- and fixed-dose regimens. Pain 115: 254-263, 2005.

10. Farrar JT, Young JP Jr, LaMoreaux L, Werth JL and Poole RM: Clinical importance of changes in chronic pain intensity measured on an 11-point numerical pain rating scale. Pain 94: 149-158, 2001.

11. Rowbotham MC: What is a 'clinically meaningful' reduction in pain? Pain 94: 131-132, 2001.

12. Cleeland CS and Ryan KM: Pain assessment: Global use of the Brief pain inventory. Ann Acad Med Singapore 23: 129-138, 1994.

13. Bonezzi C, Nava A, Barbieri M, Bettaglio R, Demartini L, Miotti D and Paulin L: Validazione della versione italiana del Brief Pain inventory nei pazienti con dolore cronico. Minerva Anestesiol 68: 607-611, 2002 (In Italian).

14. Watson CP, Moulin D, Watt-Watson J, Gordon A and Eisenhoffer J: Controlled-release oxycodone relieves neuropathic pain: A randomized controlled trial in painful diabetic neuropathy. Pain 105: 71-78, 2003.

15. Eisenberg E, McNicol ED and Carr DB: Efficacy and safety of opioid agonists in the treatment of neuropathic pain of nonmalignant origin: Systematic review and meta-analysis of randomized controlled trials. JAMA 293: 3043-3052, 2005. 